\title{
An Existence Result for a Generalized Quasilinear Schrödinger Equation with Nonlocal Term
}

\author{
Quanqing Li $\mathbb{D},{ }^{1}$ Kaimin Teng, ${ }^{2}$ Jian Zhang, ${ }^{3,4,5}$ and Jianjun Nie $\mathbb{D}^{6}$ \\ ${ }^{1}$ Department of Mathematics, Honghe University, Mengzi, Yunnan 661100, China \\ ${ }^{2}$ Department of Mathematics, Taiyuan University of Technology, Taiyuan, Shanxi 030024, China \\ ${ }^{3}$ School of Mathematics and Statistics, Hunan University of Technology and Business, Changsha, Hunan 410205, China \\ ${ }^{4}$ Key Laboratory of Hunan Province for Statistical Learning and Intelligent Computation, Hunan University of Technology \\ and Business, Changsha, Hunan 410205, China \\ ${ }^{5}$ School of Mathematics and Statistics, Central South University, Changsha, Hunan 410083, China \\ ${ }^{6}$ School of Mathematics and Physics, North China Electric Power University, Beijing 102206, China
}

Correspondence should be addressed to Jianjun Nie; niejjun@126.com

Received 29 June 2020; Revised 6 September 2020; Accepted 12 September 2020; Published 6 October 2020

Academic Editor: Mustafa Avci

Copyright (C) 2020 Quanqing Li et al. This is an open access article distributed under the Creative Commons Attribution License, which permits unrestricted use, distribution, and reproduction in any medium, provided the original work is properly cited.

In this paper, we consider the following generalized quasilinear Schrödinger equation with nonlocal term $-\operatorname{div}\left(g^{2}(u) \nabla u\right)+g(u)$ $g^{\prime}(u)|\nabla u|^{2}+V(x) u=\lambda\left[|x|^{-\mu} *|u|^{p}\right]|u|^{p-2} u, x \in \mathbb{R}^{N}$, where $N \geq 3, g: \mathbb{R} \rightarrow \mathbb{R}^{+}$is a $C^{1}$ even function, $g(0)=1, g^{\prime}(s) \geq 0$ is for all $s$ $\geq 0, \lim _{|s| \rightarrow+\infty} g(s) /|s|^{\alpha-1}:=\beta>0$ is for some $\alpha>1$, and $(\alpha-1) g(s) \geq g^{\prime}(s) s$ is for all $s \geq 0,2 \alpha \leq p \leq 2 \alpha(N-\mu) / N-2$, and $0<\mu<N$. We prove that the equation admits a solution by using a constrained minimization argument.

\section{Introduction and Preliminaries}

The main purpose of this paper is to investigate the existence of solutions for the following generalized quasilinear Schrödinger equation with nonlocal term

$$
-\operatorname{div}\left(g^{2}(u) \nabla u\right)+g(u) g^{\prime}(u)|\nabla u|^{2}+V(x) u=\lambda\left[|x|^{-\mu} *|u|^{p}\right]|u|^{p-2} u, x \in \mathbb{R}^{N},
$$

where $N \geq 3, g: \mathbb{R} \rightarrow \mathbb{R}^{+}$is a $C^{1}$ even function, $g(0)=1$, $g^{\prime}(s) \geq 0$ is for all $s \geq 0, \lim _{|s| \rightarrow+\infty} g(s) /|s|^{\alpha-1}:=\beta>0$ is for some $\alpha>1$, and $(\alpha-1) g(s) \geq g^{\prime}(s) s$ is for all $s \geq 0,2 \alpha \leq p$ $\leq 2 \alpha(N-\mu) / N-2$, and $0<\mu<N$.

When $g(u)=1$, (1) boils down to the socalled nonlinear Choquard or Choquard-Pekar equation

$$
-\Delta u+V(x) u=\lambda\left[|x|^{-\mu} *|u|^{p}\right]|u|^{p-2} u, x \in \mathbb{R}^{N} .
$$

Such like equation has several physical origins. The problem

$$
-\Delta u+u=\left[|x|^{-1} *|u|^{2}\right] u, x \in \mathbb{R}^{3}
$$

appeared at least as early as in 1954, in a work by Pekar describing the quantum mechanics of a polaron at rest [1]. In 1976, Choquard used (3) to describe an electron trapped in its own hole and in a certain approximation to HartreeFock theory of one component plasma [2]. In 1996, Penrose proposed (3) as a model of self-gravitating matter, in a program in which quantum state reduction is understood as a gravitational phenomenon [3]. In this context, equation of type (3) is usually called the nonlinear Schrödinger-Newton equation. The first investigations for existence and symmetry of the solutions to (3) go back to the works of Lieb [2] and Lions [4]. In [2], by using symmetric decreasing rearrangement inequalities, Lieb proved that the ground state solution of equation (3) is radial and unique up to translations. Lions 
[4] showed the existence of a sequence of radially symmetric solutions. Since then, many efforts have been made to study the existence of nontrivial solutions for nonlinear Choquard equations. Wei and Winter [5] showed that the ground state solution is nondegenerate. Ma and Zhao [6] considered the generalized Choquard equation

$$
-\Delta u+u=\left[|x|^{-\mu} *|u|^{q}\right]|u|^{q-2} u(q \geq 2)
$$

and proved that every positive solution of it is radially symmetric and monotone decreasing about some fixed point, under the assumption that a certain set of real numbers, defined in terms of $N, \mu$, and $q$, is nonempty. Under the same assumption, Cingolani, Clapp, and Secchi [7] gave some existence and multiplicity results in the electromagnetic case and established the regularity and some decay asymptotically at infinity of the ground states. In [8], Moroz and Van Schaftingen eliminated this restriction and showed the regularity, positivity, and radial symmetry of the ground states for the optimal range of parameters and derived decay asymptotically at infinity for them as well. Moreover, they [9] also obtained a similar conclusion under the assumption of Berestycki-Lions type nonlinearity. We point out that the existence, multiplicity, and concentration of such like equation have been established by many authors. We refer the readers to $[10,11]$ for the existence of sign-changing solutions, $[5,12]$ for the existence and concentration behavior of the semiclassical solutions and [13] for the critical nonlocal part with respect to the Hardy-Littlewood-Sobolev inequality. For more details associated with the Choquard equation, please refer to [14-16] and the references therein.

In the past, even the research on the existence of solitary wave solutions for the Schrödinger equation with local term

$-\operatorname{div}\left(g^{2}(u) \nabla u\right)+g(u) g^{\prime}(u)|\nabla u|^{2}+V(x) u=f(x, u), x \in \mathbb{R}^{N}$

is for some given special function $g(\cdot)$, see [17-19]. However, related to the nonlocal equation (1), as far as we know, there is no result in this direction. In this paper, with the aid of the new variable replacement developed by Shen and Wang in [18] and inspired by [20, 21], existence of solutions for equation (1) have been established. Problem (1) has a variational structure, and the corresponding energy functional is defined by

$$
\begin{aligned}
I(u)= & \frac{1}{2} \int_{\mathbb{R}^{N}} g^{2}(u)|\nabla u|^{2} d x+\frac{1}{2} \int_{\mathbb{R}^{N}} V(x) u^{2} d x \\
& -\frac{\lambda}{2 p} \int_{\mathbb{R}^{N}}\left[|x|^{-\mu} *|u|^{p}\right]|u|^{p} d x \\
= & \frac{1}{2} \int_{\mathbb{R}^{N}} g^{2}(u)|\nabla u|^{2} d x+\frac{1}{2} \int_{\mathbb{R}^{N}} V(x) u^{2} d x \\
& -\frac{\lambda}{2 p} \int_{\mathbb{R}^{2 N}} \frac{|u(x)|^{p}|u(y)|^{p}}{|x-y|^{\mu}} d x d y .
\end{aligned}
$$

However, $I$ is not well defined in $H^{1}\left(\mathbb{R}^{N}\right)$ because of the term $\int_{\mathbb{R}^{N}} g^{2}(u)|\nabla u|^{2} d x$. To overcome this difficulty, we make a change of variable constructed by Shen and Wang in [18]: $v:=G(u):=\int_{0}^{u} g(t) d t$. Then, we obtain

$$
\begin{aligned}
J(v)= & \frac{1}{2} \int_{\mathbb{R}^{N}}|\nabla v|^{2} d x+\frac{1}{2} \int_{\mathbb{R}^{N}} V(x) G^{-1}(v)^{2} d x \\
& -\frac{\lambda}{2 p} \int_{\mathbb{R}^{N}}\left[|x|^{-\mu} *\left|G^{-1}(v)\right|^{p}\right]\left|G^{-1}(v)\right|^{p} d x \\
= & \frac{1}{2} \int_{\mathbb{R}^{N}}|\nabla v|^{2} d x+\frac{1}{2} \int_{\mathbb{R}^{N}} V(x) G^{-1}(v)^{2} d x \\
& -\frac{\lambda}{2 p} \int_{\mathbb{R}^{2 N}} \frac{\left|G^{-1}(v(x))\right|^{p}\left|G^{-1}(v(y))\right|^{p}}{|x-y|^{\mu}} d x d y .
\end{aligned}
$$

We say that $u$ is a weak solution of (1), if

$$
\begin{aligned}
\left\langle I^{\prime}(u), \varphi\right\rangle= & \int_{\mathbb{R}^{N}}\left\{g^{2}(u) \nabla u \nabla \varphi+g(u) g^{\prime}(u)|\nabla u|^{2} \varphi\right. \\
& \left.+V(x) u \varphi-\lambda\left[|x|^{-\mu} *|u|^{p}\right]|u|^{p-2} u \varphi\right\} d x=0
\end{aligned}
$$

for all $\varphi \in C_{0}^{\infty}\left(\mathbb{R}^{N}\right)$. Let $\varphi=(1 / g(u)) \psi$. By [18], we know that the above formula is equivalent to

$$
\begin{aligned}
\left\langle J^{\prime}(v), \psi\right\rangle= & \int_{\mathbb{R}^{N}}\left\{\nabla v \nabla \psi+V(x) \frac{G^{-1}(v)}{g\left(G^{-1}(v)\right)} \psi\right. \\
& \left.-\lambda \frac{\left[|x|^{-\mu} *\left|G^{-1}(v)\right|^{p}\right]\left|G^{-1}(v)\right|^{p-2} G^{-1}(v) \psi}{g\left(G^{-1}(v)\right)}\right\} d x=0
\end{aligned}
$$

for all $\psi \in C_{0}^{\infty}\left(\mathbb{R}^{N}\right)$. Therefore, in order to find the solution of (1), it suffices to study the solution of the following equation:

$-\Delta v+V(x) \frac{G^{-1}(v)}{g\left(G^{-1}(v)\right)}-\lambda \frac{\left[|x|^{-\mu} *\left|G^{-1}(v)\right|^{p}\right]\left|G^{-1}(v)\right|^{p-2} G^{-1}(v)}{g\left(G^{-1}(v)\right)}=0$.

In this paper, we assume that the following condition holds.

$(V) V \in C\left(\mathbb{R}^{N}, \mathbb{R}\right), 0<V_{0}:=\inf _{x \in \mathbb{R}^{N}} V(x)$, and $\lim _{|x| \rightarrow \infty} V(x)=$ $+\infty$.

Set $H_{V}^{1}\left(\mathbb{R}^{N}\right)=\left\{v \in H^{1}\left(\mathbb{R}^{N}\right): \int_{\mathbb{R}^{N}}\left[|\nabla v|^{2}+V(x) v^{2}\right] d x<+\right.$ $\infty\}$ with the norm

$$
\|v\|_{H_{V}^{1}}^{2}=\int_{\mathbb{R}^{N}}\left[|\nabla v|^{2}+V(x) v^{2}\right] d x .
$$

Then, by the proof of Lemma 4 in [22], the embedding 
$H_{V}^{1}\left(\mathbb{R}^{N}\right)^{\circ} L^{t}\left(\mathbb{R}^{N}\right)$ is compact for all $t \in\left[2,2^{*}\right)$. Moreover, for any $a>0$, we define $m_{a}:=\inf _{v \in M_{a}} E(v)$, where

$$
\begin{gathered}
M_{a}:=\left\{v \in H_{V}^{1}\left(\mathbb{R}^{N}\right): \int_{\mathbb{R}^{2 N}} \frac{\left|G^{-1}(v(x))\right|^{p}\left|G^{-1}(v(y))\right|^{p}}{|x-y|^{\mu}} d x d y=a\right\} \\
E(v):=\int_{\mathbb{R}^{N}}\left[|\nabla v|^{2}+V(x) G^{-1}(v)^{2}\right] d x .
\end{gathered}
$$

Our main result is the following:

Theorem 1. Suppose that $(V)$ is satisfied, then, there exists $\lambda_{n} \rightarrow+\infty$ such that equation (1) with $\lambda=\lambda_{n}$ has a solution.

\section{Proof of Theorem 1}

To begin with, we give some lemmas.

Lemma 2 (see $[23,24]$ ). The functions $g, G$ and $G^{-1}$ possess the following properties:

(1) $G(s) \leq g(s) s \leq \alpha G(s)$ for all $s \geq 0$; $G(s) \geq g(s) s \geq \alpha G(s)$ for all $s \leq 0$

(2) $G^{-1}(s) s / g\left(G^{-1}(s)\right) \leq\left|G^{-1}(s)\right|^{2} \leq \alpha\left(G^{-1}(s) s / g\left(G^{-1}(s)\right)\right)$ for all $s \in \mathbb{R}$

(3) $|s|^{\alpha} \leq(\alpha / \beta)|G(s)|$ for all $s \in \mathbb{R}$

Proposition 3 [25] (Hardy-Littlewood-Sobolev inequality). Let $r, t>1$ and $0<\mu<N$ with $(1 / r)+(\mu / N)+(1 / t)=2$. Let $g \in L^{r}\left(\mathbb{R}^{N}\right)$ and $h \in L^{t}\left(\mathbb{R}^{N}\right)$. Then, there exists a sharp constant $C_{r, N, \mu, t}$ independent of $g$ and $h$ such that

$$
\left|\int_{\mathbb{R}^{N}} \int_{\mathbb{R}^{N}} \frac{g(x) h(y)}{|x-y|^{\mu}} d x d y\right| \leq C_{r, N, \mu, t}\|g\|_{r}\|h\|_{t} .
$$

Proof of Theorem 1. The proof consists of two steps.

Step 1: we prove that for each $a>0, m_{a}$ is achieved at some $v_{a} \in M_{a}$, which is a weak solution of equation (10) with $\lambda=\lambda_{a}$ satisfying $\lambda_{a} \in\left[\left(m_{a} / a \alpha\right),\left(\alpha m_{a} / a\right)\right]$.

For fixed $a>0$, let $\left\{v_{n}\right\} \subset M_{a}$ be a minimizing sequence for $m_{a}$, i.e., $v_{n} \in H_{V}^{1}\left(\mathbb{R}^{N}\right)$ satisfying $\int_{\mathbb{R}^{2 N}}\left(\left|G^{-1}\left(v_{n}(x)\right)\right|^{p}\right.$ $\left.\left|G^{-1}\left(v_{n}(y)\right)\right|^{p} /|x-y|^{\mu}\right) d x d y=a$ such that $E\left(v_{n}\right) \rightarrow m_{a}$ as $n$ $\rightarrow \infty$. We assert that there exists a constants $C_{1}>0$ such that $E\left(v_{n}\right) \geq C_{1} \int_{\mathbb{R}^{N}}\left[\left|\nabla v_{n}\right|^{2}+V(x) v_{n}^{2}\right] d x$. Indeed, we may assume that $v_{n} \neq 0$ (otherwise, the conclusion is trivial). If the conclusion is false, then for any positive integer $n$, we may assume that

$$
\begin{aligned}
E\left(v_{n}\right) & =\int_{\mathbb{R}^{N}}\left[\left|\nabla v_{n}\right|^{2}+V(x) G^{-1}\left(v_{n}\right)^{2}\right] d x<\frac{1}{n}\left\|v_{n}\right\|_{H_{V}^{1}}^{2} \\
& =\frac{1}{n} \int_{\mathbb{R}^{N}}\left[\left|\nabla v_{n}\right|^{2}+V(x) v_{n}^{2}\right] d x .
\end{aligned}
$$

Set $w_{n}=v_{n} /\left\|v_{n}\right\|_{H_{V}^{1}}$ and $g_{n}=G^{-1}\left(v_{n}\right)^{2} /\left\|v_{n}\right\|_{H_{V}^{1}}^{2}$. Then,

$$
\int_{\mathbb{R}^{N}}\left|\nabla w_{n}\right|^{2} d x+\int_{\mathbb{R}^{N}} V(x) g_{n}(x) d x \rightarrow 0
$$

which implies that

$\int_{\mathbb{R}^{N}}\left|\nabla w_{n}\right|^{2} d x \rightarrow 0, \int_{\mathbb{R}^{N}} V(x) g_{n}(x) d x \rightarrow 0, \int_{\mathbb{R}^{N}} V(x) w_{n}^{2} d x \rightarrow 1$

as $n \rightarrow \infty$. Then for each $\varepsilon>0$, there exists a constant $C_{2}>0$ independent of $n$ such that meas $\left(\Omega_{n}\right)<\varepsilon$, where $\Omega_{n}:=\{x \in$ $\left.\mathbb{R}^{N}:\left|G^{-1}\left(v_{n}(x)\right)\right| \geq C_{2}\right\}$. Otherwise, there exist $\varepsilon_{0}>0$ and a subsequence $\left\{G^{-1}\left(v_{n_{k}}\right)\right\}$ of $\left\{G^{-1}\left(v_{n}\right)\right\}$ such that for any positive integer $k$,

$$
\operatorname{meas}\left(\Omega_{n_{k}}\right) \geq \varepsilon_{0}>0 \text {, }
$$

where $\Omega_{n_{k}}=\left\{x \in \mathbb{R}^{N}:\left|G^{-1}\left(v_{n_{k}}(x)\right)\right| \geq k\right\}$. By $(V)$, one has

$$
\begin{aligned}
C \geq E\left(v_{n_{k}}\right) & \geq \int_{\mathbb{R}^{N}} V(x) G^{-1}\left(v_{n_{k}}(x)\right)^{2} d x \\
& \geq \int_{\Omega_{n_{k}}} V(x) G^{-1}\left(v_{n_{k}}(x)\right)^{2} d x \geq V_{0} k^{2} \varepsilon_{0} \rightarrow+\infty
\end{aligned}
$$

as $k \rightarrow+\infty$, a contradiction. Noting that as $\left|G^{-1}\left(v_{n}\right)\right|<C_{2}$, by Lemma 2 (1) and monotonicity of $g$, we have

$$
v_{n}^{2} \leq g^{2}\left(G^{-1}\left(v_{n}\right)\right) G^{-1}\left(v_{n}\right)^{2} \leq g^{2}\left(C_{2}\right) G^{-1}\left(v_{n}\right)^{2} .
$$

Hence,

$$
\int_{\mathbb{R}^{N} \backslash \Omega_{n}} V(x) w_{n}^{2} d x \leq g^{2}\left(C_{2}\right) \int_{\mathbb{R}^{N}} V(x) g_{n}(x) d x \rightarrow 0 .
$$

By the integral absolutely continuity, there exists $\varepsilon>0$ such that whenever $\Omega \subset \mathbb{R}^{N}$ and meas $(\Omega)<\varepsilon, \int_{\Omega} V(x) w_{n}^{2} d x$ $<1 / 2$. For this $\varepsilon$, one has

$$
\begin{aligned}
\int_{\mathbb{R}^{N}} V(x) w_{n}^{2} d x= & \int_{\Omega_{n}} V(x) w_{n}^{2} d x+\int_{\mathbb{R}^{N} \backslash \Omega_{n}} V(x) w_{n}^{2} d x \leq \frac{1}{2} \\
& +\int_{\mathbb{R}^{N} \Omega_{n}} V(x) w_{n}^{2} d x,
\end{aligned}
$$

which implies $1 \leq 1 / 2$, a contradiction. Therefore, up to a subsequence, there exists $v_{a} \in H_{V}^{1}\left(\mathbb{R}^{N}\right)$ such that $v_{n} \rightarrow v_{a}$ in $H_{V}^{1}\left(\mathbb{R}^{N}\right), v_{n} \rightarrow v_{a}$ in $L^{t}\left(\mathbb{R}^{N}\right)$ for $2 \leq t<2^{*}$, and $v_{n}(x)$ $\rightarrow v_{a}(x)$, a.e., on $\mathbb{R}^{N}$. By means of the definition of weak 
convergence, we know

$$
\begin{aligned}
0 \leq \int_{\mathbb{R}^{N}}\left|\nabla v_{n}-\nabla v_{a}\right|^{2} d x= & \int_{\mathbb{R}^{N}}\left|\nabla v_{n}\right|^{2} d x \\
& +\int_{\mathbb{R}^{N}}\left|\nabla v_{a}\right|^{2} d x-2 \int_{\mathbb{R}^{N}} \nabla v_{n} \cdot \nabla v_{a} d x
\end{aligned}
$$

which implies that

$\liminf _{n \rightarrow \infty} \int_{\mathbb{R}^{N}}\left|\nabla v_{n}\right|^{2} d x \geq \liminf _{n \rightarrow \infty}\left[2 \int_{\mathbb{R}^{N}} \nabla v_{n} \cdot \nabla v_{a} d x-\int_{\mathbb{R}^{N}}\left|\nabla v_{a}\right|^{2} d x\right]=\int_{\mathbb{R}^{N}}\left|\nabla v_{a}\right|^{2} d x$.

By Fatou Lemma, we have

$$
\int_{\mathbb{R}^{N}} V(x) G^{-1}\left(v_{a}\right)^{2} d x \leq \liminf _{n \rightarrow \infty} \int_{\mathbb{R}^{N}} V(x) G^{-1}\left(v_{n}\right)^{2} d x .
$$

Consequently, $\quad E\left(v_{a}\right) \leq \liminf _{n \rightarrow \infty}\left[\int_{\mathbb{R}^{N}}\left|\nabla v_{n}\right|^{2}+\int_{\mathbb{R}^{N}} V(x) G^{-1}\right.$ $\left.\left(v_{n}\right)^{2}\right]=\liminf _{n \rightarrow \infty} E\left(v_{n}\right)$. Moreover, by the Hardy-LittlewoodSobolev inequality and Lemma 2 (3), one has

$$
\begin{aligned}
a= & \int_{\mathbb{R}^{2 N}} \frac{\left|G^{-1}\left(v_{n}(x)\right)\right|^{p}\left|G^{-1}\left(v_{n}(y)\right)\right|^{p}}{|x-y|^{\mu}} d x d y \\
& \leq C\left\|\left|G^{-1}\left(v_{n}\right)\right|^{p}\right\|_{2 N / 2 N-\mu}^{2} \leq C\left(\int_{\mathbb{R}^{N}}\left|v_{n}\right|^{2 N p / \alpha(2 N-\mu)} d x\right)^{2 N-\mu / N} .
\end{aligned}
$$

Since $2<2 N p / \alpha(2 N-\mu)<2^{*}$, by Lemma A.1 in [26] and Lebesgue's dominated convergence theorem, we can easily infer that $\int_{\mathbb{R}^{2 N}}\left(\left|G^{-1}\left(v_{a}(x)\right)\right|^{p}\left|G^{-1}\left(v_{a}(y)\right)\right|^{p} /|x-y|^{\mu}\right) d$ $x d y=a$, and so $v_{a} \in M_{a}$. Hence, $m_{a} \leq E\left(v_{a}\right) \leq \liminf _{n \rightarrow \infty} E\left(v_{n}\right)$ $=m_{a}$, which means that $m_{a}$ is achieved at some $v_{a} \in M_{a}$. Moreover, by a standard argument, we can conclude that $v_{a}$ is a weak solution of

$-\Delta v+V(x) \frac{G^{-1}(v)}{g\left(G^{-1}(v)\right)}=\lambda_{a} \frac{\left[|x|^{-\mu} *\left|G^{-1}(v)\right|^{p}\right]\left|G^{-1}(v)\right|^{p-2} G^{-1}(v)}{g\left(G^{-1}(v)\right)}$.

Multiplying the above equation by $v_{a}$ and integrating over $\mathbb{R}^{N}$, one has

$$
\begin{aligned}
\int_{\mathbb{R}^{N}}\left|\nabla v_{a}\right|^{2} d x+ & \int_{\mathbb{R}^{N}} V(x) \frac{G^{-1}\left(v_{a}\right) v_{a}}{g\left(G^{-1}\left(v_{a}\right)\right)} d x \\
& =\lambda_{a} \int_{\mathbb{R}^{N}} \frac{\left[|x|^{-\mu} *\left|G^{-1}\left(v_{a}\right)\right|^{p}\right]\left|G^{-1}\left(v_{a}\right)\right|^{p-2} G^{-1}\left(v_{a}\right) v_{a}}{g\left(G^{-1}\left(v_{a}\right)\right)} d x .
\end{aligned}
$$

By Lemma 2 (2), we obtain $m_{a} / a \alpha \leq \lambda_{a} \leq \alpha m_{a} / a$. Indeed, by Lemma 2 (2), we have

$$
\begin{aligned}
\frac{1}{\alpha} m_{a} & =\frac{1}{\alpha} E\left(v_{a}\right)=\frac{1}{\alpha}\left[\int_{\mathbb{R}^{N}}\left|\nabla v_{a}\right|^{2} d x+\int_{\mathbb{R}^{N}} V(x) G^{-1}\left(v_{a}\right)^{2} d x\right] \\
& \leq \int_{\mathbb{R}^{N}}\left|\nabla v_{a}\right|^{2} d x+\frac{1}{\alpha} \int_{\mathbb{R}^{N}} V(x) G^{-1}\left(v_{a}\right)^{2} d x \\
& \leq \int_{\mathbb{R}^{N}}\left|\nabla v_{a}\right|^{2} d x+\int_{\mathbb{R}^{N}} V(x) \frac{G^{-1}\left(v_{a}\right) v_{a}}{g\left(G^{-1}\left(v_{a}\right)\right)} d x \\
& \leq \lambda_{a} \int_{\mathbb{R}^{N}}\left[|x|^{-\mu} *\left|G^{-1}\left(v_{a}\right)\right|^{p}\right]\left|G^{-1}\left(v_{a}\right)\right|^{p} d x=\lambda_{a} \cdot a,
\end{aligned}
$$

i.e., $\lambda_{a} \geq m_{a} / a \alpha$. Furthermore,

$$
\begin{aligned}
m_{a} & =E\left(v_{a}\right)=\int_{\mathbb{R}^{N}}\left|\nabla v_{a}\right|^{2} d x+\int_{\mathbb{R}^{N}} V(x) G^{-1}\left(v_{a}\right)^{2} d x \\
& \geq \int_{\mathbb{R}^{N}}\left|\nabla v_{a}\right|^{2} d x+\int_{\mathbb{R}^{N}} V(x) \frac{G^{-1}\left(v_{a}\right) v_{a}}{g\left(G^{-1}\left(v_{a}\right)\right)} d x \\
& \geq \frac{\lambda_{a}}{\alpha} \int_{\mathbb{R}^{N}}\left[|x|^{-\mu} *\left|G^{-1}\left(v_{a}\right)\right|^{p}\right]\left|G^{-1}\left(v_{a}\right)\right|^{p} d x=\frac{\lambda_{a}}{\alpha} \cdot a,
\end{aligned}
$$

i.e., $\lambda_{a} \leq \alpha m_{a} / a$.

Step 2: we prove that $\lambda_{a} \rightarrow+\infty$ as $a \rightarrow 0$.

If the conclusion is false, then there exists a constant $G_{0}$ $>0$ and $a_{n} \rightarrow 0(n \rightarrow \infty)$ such that $\lambda_{n}:=\lambda_{a_{n}} \leq G_{0}$. Set $v_{n}:=$ $v_{a_{n}}$, by Lemma 2 (2) and Hardy-Littlewood-Sobolev inequality, we have

$$
\begin{aligned}
\int_{\mathbb{R}^{N}}\left|\nabla v_{n}\right|^{2} d x+ & \int_{\mathbb{R}^{N}} V(x) \frac{G^{-1}\left(v_{n}\right) v_{n}}{g\left(G^{-1}\left(v_{n}\right)\right)} d x \\
& =\lambda_{n} \int_{\mathbb{R}^{N}} \frac{\left[|x|^{-\mu} *\left|G^{-1}\left(v_{n}\right)\right|^{p}\right]\left|G^{-1}\left(v_{n}\right)\right|^{p-2} G^{-1}\left(v_{n}\right) v_{n}}{g\left(G^{-1}\left(v_{n}\right)\right)} d x \\
& \leq \lambda_{n} \int_{\mathbb{R}^{N}}\left[|x|^{-\mu} *\left|G^{-1}\left(v_{n}\right)\right|^{p}\right]\left|G^{-1}\left(v_{n}\right)\right|^{p} d x=\lambda_{n} a_{n} \\
& \leq G_{0} \cdot a_{n} \rightarrow 0
\end{aligned}
$$

as $n \rightarrow \infty$. Since $2<2 N p / \alpha(2 N-\mu)<2^{*}$, there exists a constant $\theta \in(0,1)$ such that $1 / 2 N p / \alpha(2 N-\mu)=(\theta / 2)+(1-\theta /$ $\left.2^{*}\right)$. Consequently, by Lemma $2(3),(V)$, Hölder inequality, 
and Young inequality, one has

$$
\begin{aligned}
\int_{\mathbb{R}^{N}}\left|G^{-1}\left(v_{n}\right)\right|^{2 N p / 2 N-\mu} d x \leq & C \int_{\mathbb{R}^{N}}\left|v_{n}\right|^{2 N p / \alpha(2 N-\mu)} d x \\
= & C \int_{\mathbb{R}^{N}}\left|v_{n}\right|^{\theta 2 N p / \alpha(2 N-\mu)}\left|v_{n}\right|^{(1-\theta) 2 N p / \alpha(2 N-\mu)} d x \\
\leq & C\left\|v_{n}\right\|_{2}^{\theta 2 N p / \alpha(2 N-\mu)}\left\|v_{n}\right\|_{2^{*}}^{(1-\theta) 2 N p / \alpha(2 N-\mu)} \\
\leq & C \theta\left\|v_{n}\right\|_{2}^{2 N p / \alpha(2 N-\mu)} \\
& +C(1-\theta)\left\|v_{n}\right\|_{2^{*}}^{2 N p / \alpha(2 N-\mu)} \\
\leq & C\left(\int_{\mathbb{R}^{N}} V(x) v_{n}^{2} d x\right)^{N p / \alpha(2 N-\mu)} \\
& +C\left(\int_{\mathbb{R}^{N}}\left|\nabla v_{n}\right|^{2} d x\right)^{N p / \alpha(2 N-\mu)} \\
\leq & C\left(\int_{\mathbb{R}^{N}}\left[\left|\nabla v_{n}\right|^{2}+V(x) v_{n}^{2}\right] d x\right)^{N p / \alpha(2 N-\mu)} \\
\leq & C\left(\int_{\mathbb{R}^{N}}\left[\left|\nabla v_{n}\right|^{2}+V(x) G^{-1}\left(v_{n}\right)^{2}\right] d x\right)^{N p / \alpha(2 N-\mu)}
\end{aligned}
$$

Hence, again, by Lemma 2 (2) and Hardy-LittlewoodSobolev inequality, we have

$$
\begin{aligned}
\int_{\mathbb{R}^{N}}\left|\nabla v_{n}\right|^{2} d x+ & \int_{\mathbb{R}^{N}} V(x) \frac{G^{-1}\left(v_{n}\right) v_{n}}{g\left(G^{-1}\left(v_{n}\right)\right)} d x \\
& \leq \lambda_{n} \int_{\mathbb{R}^{N}}\left[|x|^{-\mu} *\left|G^{-1}\left(v_{n}\right)\right|^{p}\right]\left|G^{-1}\left(v_{n}\right)\right|^{p} d x \\
& \leq C \lambda_{n}\left\|\left|G^{-1}\left(v_{n}\right)\right|^{p}\right\|_{2 N / 2 N-\mu}^{2} \\
& \leq C \cdot G_{0}\left(\int_{\mathbb{R}^{N}}\left[\left|\nabla v_{n}\right|^{2}+V(x) G^{-1}\left(v_{n}\right)^{2}\right] d x\right)^{p / \alpha} \\
& \leq C \cdot G_{0}\left(\int_{\mathbb{R}^{N}}\left[\left|\nabla v_{n}\right|^{2}+V(x) \frac{G^{-1}\left(v_{n}\right) v_{n}}{g\left(G^{-1}\left(v_{n}\right)\right)}\right] d x\right)^{p / \alpha},
\end{aligned}
$$

and so $\int_{\mathbb{R}^{N}}\left[\left|\nabla v_{n}\right|^{2}+V(x)\left(G^{-1}\left(v_{n}\right) v_{n} / g\left(G^{-1}\left(v_{n}\right)\right)\right)\right] d x \geq C$ since $p / \alpha \geq 2>1$, a contradiction. By steps 1 and 2, we complete the proof of Theorem 1 .

\section{Data Availability}

No data were used to support this study.

\section{Conflicts of Interest}

The authors declare that they have no conflicts of interest.

\section{Acknowledgments}

This work is supported in part by the National Natural Science Foundation of China (Nos. 11801153, 11801545, 11701322, and 11901514), the Yunnan Province Applied Basic Research for Youths (No. 2018FD085), the Yunnan Province Local University (Part) Basic Research Joint Project (No. 2017FH001-013), the Yunnan Province Applied Basic Research for General Project (No. 2019FB001), and Technology Innovation Team of University in Yunnan Province.

\section{References}

[1] S. Pekar, Untersuchungen über die Elektronentheorie der Kristalle, Akademie Verlag, Berlin, Germany, 1954.

[2] E. H. Lieb, "Existence and uniqueness of the minimizing solution of Choquard's nonlinear equation," Studies in Applied Mathematics, vol. 57, no. 2, pp. 93-105, 1977.

[3] R. Penrose, "On gravity's role in quantum state reduction," General Relativity and Gravitation, vol. 28, no. 5, pp. 581600, 1996.

[4] P. L. Lions, "The Choquard equation and related questions," Nonlinear Analysis, vol. 4, no. 6, pp. 1063-1072, 1980.

[5] J. Wei and M. Winter, "Strongly interacting bumps for the Schrödinger-Newton equations," Journal of Mathematical Physics, vol. 50, no. 1, article 012905, 2009.

[6] L. Ma and L. Zhao, "Classification of positive solitary solutions of the nonlinear Choquard equation," Archive for Rational Mechanics and Analysis, vol. 195, no. 2, pp. 455-467, 2010.

[7] S. Cingolani, M. Clapp, and S. Secchi, "Multiple solutions to a magnetic nonlinear Choquard equation," Zeitschrift für Angewandte Mathematik und Physik, vol. 63, no. 2, pp. 233-248, 2012.

[8] V. Moroz and J. Van Schaftingen, "Groundstates of nonlinear Choquard equations: existence, qualitative properties and decay asymptotics," Journal of Functional Analysis, vol. 265, no. 2, pp. 153-184, 2013.

[9] V. Moroz and J. Van Schaftingen, "Existence of groundstates for a class of nonlinear Choquard equations," Transactions of the American Mathematical Society, vol. 367, no. 9, pp. 6557-6579, 2015.

[10] M. Clapp and D. Salazar, "Positive and sign changing solutions to a nonlinear Choquard equation," Journal of Mathematical Analysis and Applications, vol. 407, no. 1, pp. 1-15, 2013.

[11] M. Ghimenti and J. Van Schaftingen, "Nodal solutions for the Choquard equation," Journal of Functional Analysis, vol. 271, no. 1, pp. 107-135, 2016.

[12] V. Moroz and J. Van Schaftingen, "Semi-classical states for the Choquard equation," Calculus of Variations and Partial Differential Equations, vol. 52, no. 1-2, pp. 199-235, 2015.

[13] V. Moroz and J. Van Schaftingen, "Groundstates of nonlinear Choquard equations: Hardy-Littlewood-Sobolev critical exponent," Communications in Contemporary Mathematics, vol. 17, no. 5, article 1550005, 2015.

[14] D. Cassani and J. Zhang, "Choquard-type equations with Hardy-Littlewood-Sobolev upper-critical growth," Advances in Nonlinear Analysis, vol. 8, pp. 1184-1212, 2018.

[15] Q. Li, K. Teng, and J. Zhang, "Ground state solutions for fractional Choquard equations involving upper critical exponent," Nonlinear Analysis, vol. 197, p. 111846, 2020.

[16] J. Seok, "Limit profiles and uniqueness of ground states to the nonlinear Choquard equations," Advances in Nonlinear Analysis, vol. 8, no. 1, pp. 1083-1098, 2018.

[17] N. S. Papageorgiou, V. D. Radulescu, and D. D. Repovs, Nonlinear Analysis-Theory and Methods, Springer Monographs in Mathematics, Springer, Cham, Switzerland, 2019.

[18] Y. Shen and Y. Wang, "Soliton solutions for generalized quasilinear Schrödinger equations," Nonlinear Analysis, vol. 80, pp. 194-201, 2013.

[19] Y. Xue and C. Tang, "Existence of a bound state solution for quasilinear Schrödinger equations," Advances in Nonlinear Analysis, vol. 8, pp. 323-338, 2017. 
[20] J. Liu and Z. Wang, "Soliton solutions for quasilinear Schrödinger equations, I," Proceedings of the American Mathematical Society, vol. 131, pp. 441-448, 2003.

[21] Q. Li and X. Wu, "Soliton solutions for fractional Schrödinger equations," Applied Mathematics Letters, vol. 53, pp. 119-124, 2016.

[22] W. Zou and M. Schechter, Critical Point Theory and its Applications, Springer, New York, NY, USA, 2006.

[23] Y. Deng, S. Peng, and S. Yan, "Critical exponents and solitary wave solutions for generalized quasilinear Schrödinger equations," Journal of Differential Equations, vol. 260, no. 2, pp. 1228-1262, 2016.

[24] Q. Li and X. Wu, "Existence, multiplicity, and concentration of solutions for generalized quasilinear Schrödinger equations with critical growth," Journal of Mathematical Physics, vol. 58, no. 4, article 041501, 2017.

[25] E. H. Lieb and M. Loss, Analysis, American Mathematical Society, Providence, RI, USA, 2nd edition, 2001.

[26] M. Willem, Minimax Theorem, Birkhäuser Boston, Inc., Boston, MA, USA, 1996. 